\title{
Automatic Resolution of Object Features from Engineering Drawings for 3D Reconstruction
}

\author{
Miri Weiss and Dov Dori \\ Faculty of Industrial Engineering and Management \\ Technion, Israel Institute of Technology \\ Haifa 32000, Israel \\ e-mail: \{iemiri, dori\} @ie.technion.ac.il
}

\begin{abstract}
In spite of the fact that 3D objects reconstruction from 2D views is an intensively researched problem, no comprehensive satisfactory solution has been found. This work contributes towards automating the 3D reconstruction by enabling automatic definition of features of planes in 2D orthographic projections. To this end, we introduce the concept of loops in 2D views and their states, which are topology/line continuity attribute value combinations. Correspondence is established between a loop's state and its content attribute value, which may be matter, protrusion or depression. Ambiguous cases require interrogation of complementary views, as we demonstrate in a few examples. This work is designed to be incorporated as part of the Machine Drawing Understanding System, which is aimed at recognition and interpretation of engineering drawings.
\end{abstract}

\section{Introduction}

A number of approaches and algorithms have been developed over the past two decades to automatically interpret a set of user-supplied orthographic views for the purpose of 3D object reconstruction. The two main reconstruction approaches are the wireframeoriented bottom up approach and the volume-oriented approach. Wireframe-oriented bottom up algorithms $[3,9,10,11,12,15,17]$ are based on four major steps: (1) transformation of $2 \mathrm{D}$ vertices extracted from orthographic views into $3 \mathrm{D}$ vertices, (2) generation of $3 \mathrm{D}$ edges from the $3 \mathrm{D}$ vertices, (3) construction of faces from the $3 \mathrm{D}$ edges, and (4) formation of 3D objects from the 3D faces.

Volume-oriented algorithms are based on constructing 3D primitive subparts through translation sweep operations and combining them into a complete object. Aldefeld [1] suggested to view a complex part as a composition of elementary objects belonging to a set of predefined classes. Recognition of these objects is done by making use of knowledge about the class-dependent pattern of their $2 \mathrm{D}$ representation. In further work [2], Aldefeld and Richter suggested to interpret a 3D body from its $2 \mathrm{D}$ projections by a user-guided process, while using automatic procedures where they can be relied upon. Nagasami and Noshir [14] extended and implemented [2] by defining two classes of objects that represent a wide verity of objects. One class is obtained by translation sweep operations with a planar base, an arbitrary contour in one view and a uniform thickness in the direction perpendicular to the base. The other class consists of objects having an axis of symmetry. Chen and Perng [5] introduced a method for reconstructing 3-D objects, which may be polyhedrons, cylinders, partial cylinders and their composites. The algorithm consists of three stages: (1) decomposition, in which the input drawing is decomposed into several predefined types of subviews, (2) reconstruction, in which translational sweep and cutting operations are used to reconstruct the corresponding subpart of each set of subviews, and (3) composition, in which volume enclosure relations among various subparts are utilized to compose the complete object, which is represented by a CSG tree.

Volume-oriented algorithms follow human intuition of forming solid primitives and combining them into a 3D solid object from its 2D orthographic views. Nevertheless, 
most approaches involve a user guided process as a starting point for recognition of basic primitives in the 2D orthographic engineering drawing. Meeran and Pratt [13] proposed a method of automated feature recognition from 2D drawings based on determining the 'seed' entity for each of the three views. Starting from the seed, chains of connected entities are built up by following adjacent entities in the periphery of the ones already detected in one view or more.

While most volume- oriented approaches require that a human first points at certain areas in the view and indicates whether they are matter or protrusion or depression (hole), the work presented in this paper enables this task to be carried out automatically. From this point on, one can continue the automatic procedure of existing volume- oriented approaches.

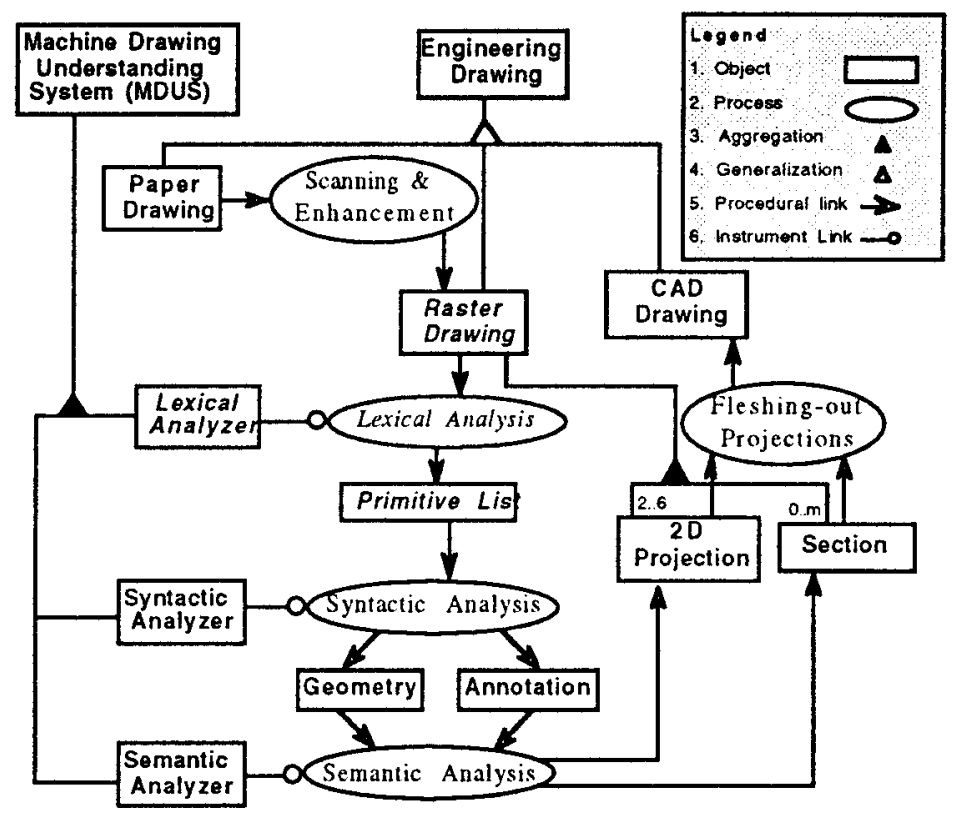

Fig 1. An Object Process Diagram (OPD) of MDUS and its environment

Our work is aimed at devising an automatic $3 \mathrm{D}$ reconstruction procedure embedded as the 3D semantic level of the Machine Drawing Understanding System (MDUS) [6]. MDUS is an experimental system designed to automate the process of converting mechanical engineering drawings into $\mathrm{CAD}$ format.

Figure 1 is an object-process diagram (OPJ) [8] of MDUS and its environment. Objects and processes are denoted by boxes and ellipses, respectively, where structural relations (aggregation and generalization) and procedural relations (links between objects and processes) are explicitly shown. MDUS consists of three parts: Lexical Analyzer [6], Syntactic Analyzer [7,16], and Semantic Analyzer. The Lexical Analysis process results in Primitive Lists which contain bars (straight line segments with non-zero width), arcs, arrowheads and textboxes. These primitives are processed by the Syntactic Analyzer in the Syntactic Analysis process, in which geometry primitives are separated from the annotation ones. In the Semantic Analysis process, these objects are used to carry out 2D understanding, i.e., understanding the drawing at the projection (orthographic view) level. 
The process of automated feature recognition from the 2D drawing is performed next, and $3 \mathrm{D}$ reconstruction is the last major semantic phase. This final process produces the CAD drawing, which is a complete $3 \mathrm{D}$ representation of the object described in the annotated set of $2 \mathrm{D}$ views depicted in the original paper drawing.

\section{Basic definitions}

An engineering drawing is a combination of a number (usually three) of orthographic views of an object, showing projections of the object's boundary, using lines with two continues value-types solid and dashed, Symbolic representations of features (e.g., radius of a hole), representations of machining requirements (e.g., surface finish), dimensions, tolerances and textual annotations. The lexical analysis of MDUS involves recognition of solid and dashed wires (bars and arcs), while the syntactic and semantic phases determine which wire is a geometry wire and which is an annotation wire. These classified primitives, and in particular the geometry ones, are the input to the 3D reconstruction process. We define and use the following definitions in our work.

Contour - the ordered collection of the outmost closed chain of wires.

Loop - a minimal set of closed consecutive wires (bars and/or arcs), where closed means that the starting vertex of the first wire coincides with the ending vertex of the last wire.

A loop is characterized by three attributes: topology, continuity and contents. We examine the possible values of each one of these attributes next.

The possible values of the loop's topology attribute

The loop's topology pertains to the planar position of the loop relative to other loops in the view. Possible values are contour, internal, external and intermediate, which are defined as follows.

Internal loop - a loop in which all wires are internal i.e., each wire is inside the area surrounded by the contour or a loop within it, and no wire has a common edge with any wire of an other loop.

External loop - a loop which has at least one wire that coincides with a wire of the contour.

Intermediate loop - a loop which is neither internal nor external.

Figure 2 is a $2 \mathrm{D}$ front view of a 3D object described in an engineering drawing, which contains solid and dashed wires, and in which the contour is marked by a thick line. Figure 3 exemplifies the definition of loops and their topology values. Each of the six loops is depicted separately.

The possible values of a loop's continuity attribute

Any wire of the loop can be solid (continuos) or dashed (discontinuous). This gives rise to three loop continuity values: solid, dashed, and mixed, as follows.

solid loop - a loop consisting of solid wires only.

dashed loop - a loop consisting of dashed wires only.

mixed loop - a loop consisting of a mixture of at least one solid wire and one dashed wire.

\section{The possible values of a loop's content attribute}

The loop's content pertains to the semantics of what the loop symbolizes, i.e., the presence of matter or lack thereof. The possible values are matter, depression, protrusion and undefined as follows. 


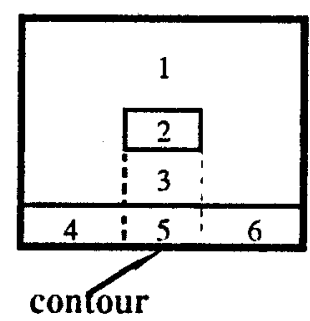

Fig. 2. Original $2 \mathrm{D}$ view

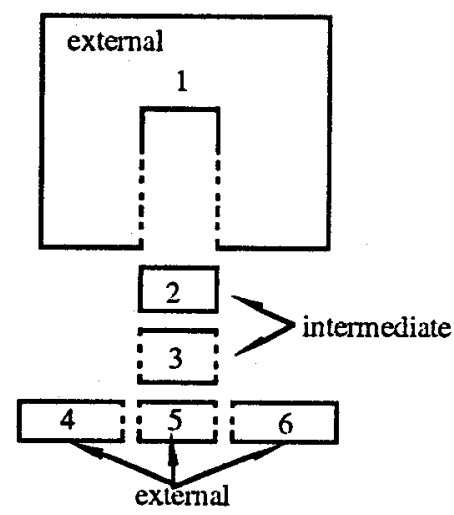

Fig. 3. Loops in the original $2 \mathrm{D}$ view

matter loop - a loop enclosing solid matter of the 3D object

depression loop - a loop enclosing a through (piercing) hole or a lowered surface, i.e., a surface which is located below the matter surrounding loop surface level in the 3D object. protrusion loop - a loop enclosing a heightened surface, i.e., a surface which is located above the surrounding matter loop surface level in the 3D object.

undefined loop - a loop whose content is not defined.

\section{Approach and Algorithm}

Our proposed approach for $3 \mathrm{D}$ object feature recognition in engineering drawing is based on the following five stages:

(1) finding all vertices in the $2 \mathrm{D}$ orthographic view;

(2) generating all possible loops;

(3) determining the loops' topology and continuity attribute values;

(4) determining the loops' content attribute value; and

(5) validation and extension of loops' contents.

The details of each step follow.

Step 1: Finding all verices in the 2D orthographic view

The $2 \mathrm{D}$ orthographic views are analyzed for defining all vertices connecting or intersecting bars and arcs in the drawing [13]. The formulation of bar intersection is given below. Arc intersection is described in detail in [4].

Consider two line segments $\mathrm{KL}$ and $\mathrm{MN}$ whose parametric equations are listed in Equations (1) and (2), respectively.

$$
\begin{aligned}
& \begin{cases}x=x_{\mathrm{K}}+\left(x_{\mathrm{L}}-x_{\mathrm{K}}\right) s & 0 \leq s \leq 1 \\
y=y_{\mathrm{K}}+\left(y_{\mathrm{L}}-y_{\mathrm{K}}\right) s & \end{cases} \\
& \begin{cases}x=x_{\mathrm{M}}+\left(x_{\mathrm{N}}-x_{\mathrm{M}}\right) t & 0 \leq t \leq 1 \\
y=y_{\mathrm{M}}+\left(y_{\mathrm{N}}-y_{\mathrm{M}}\right) t & \end{cases}
\end{aligned}
$$


The parameter $s$ of $\mathrm{KL}$ runs from 0 at $\mathrm{K}$ to 1 at $\mathrm{L}$, and the parameter $t$ of $\mathrm{MN}$ runs from 0 at $M$ to 1 at $N$. The solution of the set of simultaneous equations (1) and (2), listed in Equation (3), is the bars intersection point.

$$
\left\{\begin{array}{c}
s=\frac{\left(x_{N}-x_{M}\right)\left(y_{M}-y_{K}\right)-\left(y_{N}-y_{M}\right)\left(x_{M}-x_{K}\right)}{\left(x_{N}-x_{M}\right)\left(y_{L}-y_{K}\right)-\left(y_{N}-y_{M}\right)\left(x_{L}-x_{K}\right)} \\
t=\frac{\left(x_{L}-x_{K}\right)\left(y_{M}-y_{K}\right)-\left(y_{L}-y_{K}\right)\left(x_{M}-x_{K}\right)}{\left(x_{N}-x_{M}\right)\left(y_{L}-y_{K}\right)-\left(y_{N}-y_{M}\right)\left(x_{L}-x_{K}\right)}
\end{array}\right.
$$

If $s \in[0,1]$ and $t \in[0,1]$, i.e., the values of both parameters are within the 0 to 1 range, then the intersection point of the two segments lies along each one of them (rather than on their extensions). The values of the intersection point are given in Equation (4).

$$
\begin{aligned}
& x=x_{\mathrm{K}}+\left(x_{\mathrm{L}}-x_{\mathrm{K}}\right) s=x_{\mathrm{M}}+\left(x_{\mathrm{N}}-x_{\mathrm{M}}\right) t \\
& y=y_{\mathrm{K}}+\left(y_{\mathrm{L}}-y_{\mathrm{K}}\right) s=y_{\mathrm{M}}+\left(y_{\mathrm{N}}-y_{\mathrm{M}}\right) t
\end{aligned}
$$

The points found in this process are added to the list of $2 \mathrm{D}$ vertices which is kept separately for each 2D orthographic view.

\section{Step 2: Generating all possible loops}

Based on the definition of a loop as a minimal closed consecutive set of wires, loops are determined by following the turn-to-leftmost rule [9]. A loop generation process starts from an arbitrary wire and traverses in a predefined direction to the next wire. The turn-tothe-lefunost rule implies that if there is more than one wire at any vertex, the wire which makes the smallest angle with the current wire is chosen to continue the chain. A loop is defined and is complete when the starting wire is traversed in the same direction for the second time. The loop generation procedure in the $2 \mathrm{D}$ orthographic view is complete when all possible loops have been found.

\section{Step 3: determining the loops' topology and continuity attribute values}

Each loop found in the previous step has a certain value for it topology attribute and a certain value for its continuity attribute. The Cartesian product of the two attribute value sets yields nine loop types. Each type features selective multiple inheritance: it inherits one continuity value and one topology value. Figure 4 shows the state of each one of the six loops, where the state is defined as the tuple \{topology value, continuity value\}. Using this definition, the state of loop 1 in Figure 4 is \{external,mixed\}, the state of loop 2 is (intermediate, solid\}, the state of loop 3 is \{intermediate, mixed\}, and the states of each one of the loops $4,5,6$ is \{external, mixed .

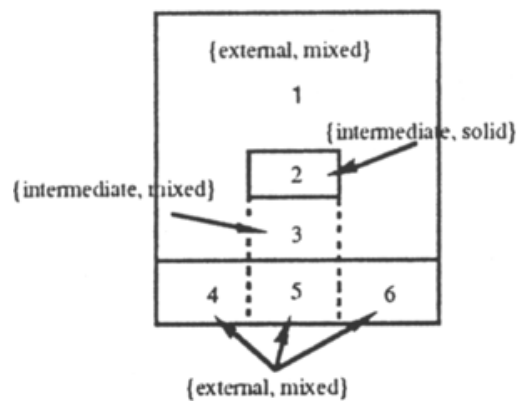

Fig. 4. The topology and continuity loops of the values in Fig. 2

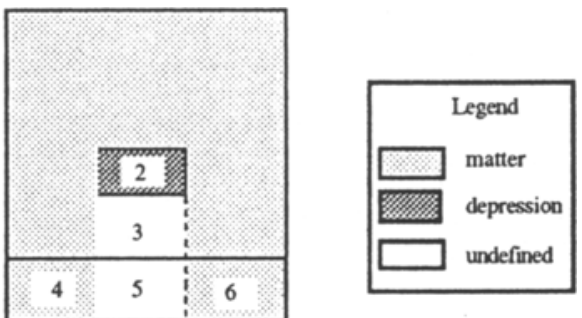

Fig. 5. The content value of the loops in Fig. 4 
Step 4: determining the loop's content attribute value.

Having determined the topology and continuity values of each loop, it is now possible to determine the loop's content (feature). Figure 5 denotes by gray levels the three possible content values that correspond to the different states (topology - continuity pairs). The correspondence rules are listed in Table 1.

Consider, for example, loop 1, whose state is \{external, mixed\}. The entry for external and mixed in Table 1 has a condition. Since at least two adjacent wires coincide with the contour, the condition is satisfied, hence we conclude that the loop represents matter.

\begin{tabular}{|c|c|c|c|}
\hline $\begin{array}{c}\text { topology } \\
\text { continuity }\end{array}$ & internal & \multicolumn{1}{|c|}{ external } & \multicolumn{1}{|c|}{ intermediate } \\
\hline solid & $\begin{array}{c}\text { depression or } \\
\text { protrusion }\end{array}$ & matter, depression or protrusion & \multicolumn{1}{c|}{$\begin{array}{c}\text { depression or } \\
\text { protrusion }\end{array}$} \\
\hline dashed & undefined & non-existing & \multicolumn{1}{|c|}{ undefined } \\
\hline mixed & non-existing & $\begin{array}{l}\text { if at least 2 adjacent solid wires } \\
\text { coincide with } \\
\text { the contour loop -->matter } \\
\text { else }\end{array}$ & $\begin{array}{l}\text { if at least 2 solid wires } \\
\text { adjacent -->matter } \\
\text { else } \quad-\rightarrow \text { undefined }\end{array}$ \\
\hline
\end{tabular}

Table 1: Loop content of the various loop states (topology / continuity combinations).

Step 5 :Validation and extension of loops' contents.

The validation and extension process of step 4 is performed by relating two or more $2 \mathrm{D}$ view to provide more information where needed. Loop 2 in Figure 5, is defined in step 4 as 'depression or protrusion'. By combining the front view ( $\mathrm{z}-\mathrm{x}$ plane) and the side view ( $\mathrm{z}$ $y$ plane), the choice between the two options is clear. This content value determination process is performed for each loop where Table 1 yields more than one constant value or 'undefined'. Another example is the case of a loop whose state is \{external, solid\}. The corresponding entry in Table 1 is 'matter', 'depression' or 'protrusion'. Since the value cannot be determined definitely from one $2 \mathrm{D}$ orthographic view, information from an additional view is needed.

The validation and extension of the loop's contents consists of two stages. The first stage is the determination of the loop's content in case of multiple solution or 'undefined' content. The second stage is the utilization of each loop in each one of the 2D orthographic views for a complete 3D representation. Meerean and Pratt [13] isolated simple features in a 2D drawing and defined 'patterns' for representations of holes, slots, pockets, etc., in three $2 \mathrm{D}$ drawings.

Figure 6 is an example from [13] of a pattern for a cylindrical through hole. The pattern in the top view is a circle, while in the front and side views the bole is a rectangle with two solid and two dashed wires. These contour lines of the circle (top view) are represented as dashed lines in the front and side views.

In our work the state of each such loop is \{external, mixed\}. According to Table 1, the content of such loop is undefined. Each one of these two loops correspond to the circle in the top view. The state of the circle loop is \{internal, solid\}, which according to Table 1 is a depression. Moreover, since the dashed lines connect the top and the bottom lines (representing the top and bottom planes) we can deduce that the depression is a through hole. To provide an exhaustive list of cases, a full analysis of the possible combinations of interactions among the various views must be carried out. This work is currently under way. 

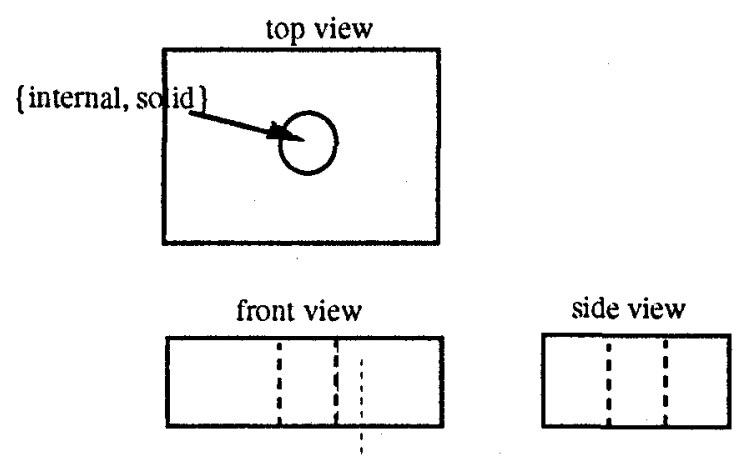

Fig. 6. Pattern for cylindrical through hole [13].

\section{Examples}

Figures 7 and 8 are simple examples which describe the top, front and side views of a 3D object. The loops are indicated by their states, i.e., their ftopology value, continuity value pairs found by implementing steps 2,3 and 4 in the algorithm. The gray levels denote the possible content values. As described in the legend, the loop's content attribute value may be matter, depression or undefined.
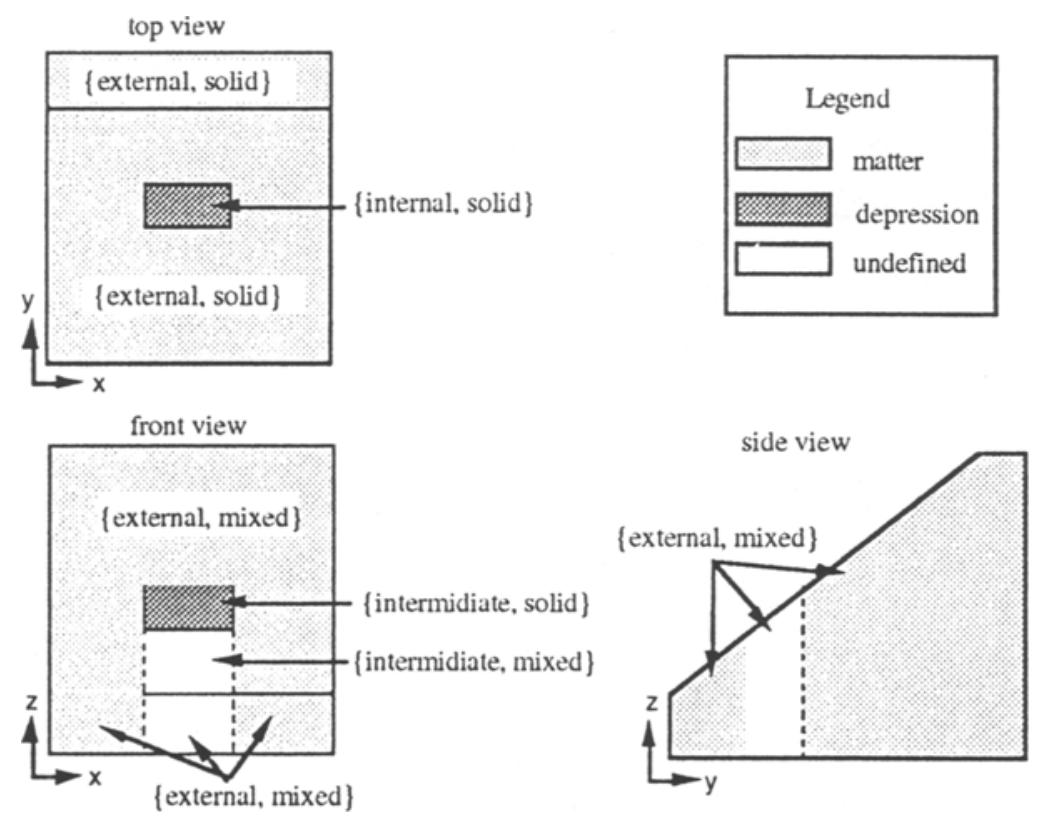

Fig.7. Three orthographic views of a 3D object with topology, continuity and content of the loops. 

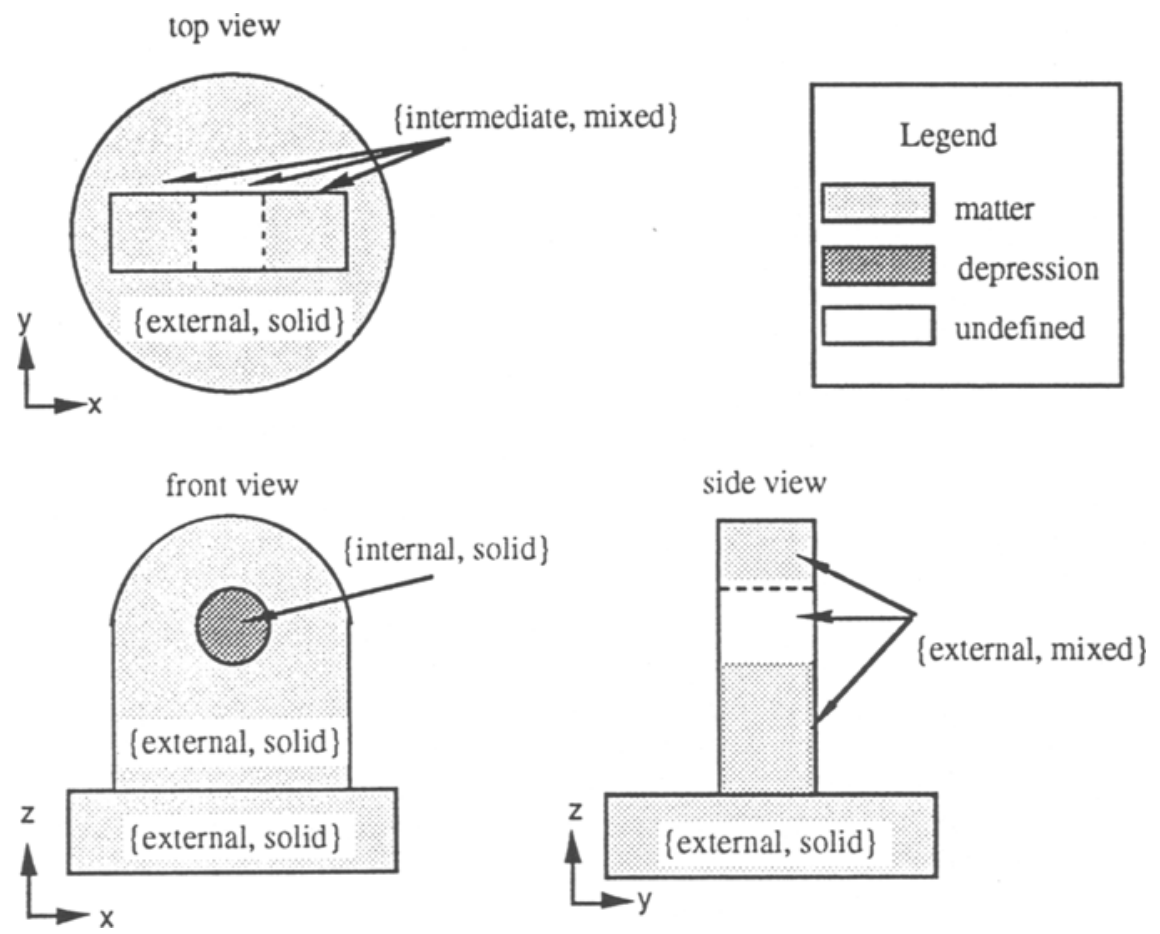

Fig. 8. Three orthographic views of a 3D object with topology, continuity and content of the loops.

\section{Conclusions and further research}

We have proposed a method for determining the features of an object which is represented by three orthographic views. The method is based on definition of a loop as the minimal set of consecutive wires in a view. Determining the content value (feature) of each as matter, protrusion or depression is a function of the loop's state, i.e., its topology/continuity attribute value combination. The method is successfully demonstrated on examples but obviously there are more complex cases, the resolution of which would require exhaustive elaboration of all the possible combinations and interactions among loops in different views. As noted, research on these complex interactions is being carried out, and we expect to be able to characterize at least all the typical cases found in engineering drawings that describe mechanical parts low to medium complexity.

\section{References:}

1. Aldefeld, B., On automatic recognition of 3D structures from $2 \mathrm{D}$ representations, CAD, Vol. 15, No. 2, 1983.

2. Aldefeld, B. and Richter H., Semiautomatic three dimensional interpretation of line drawings, Computers and Graphics, Vol. 8, No. 4, pp. 371-380, 1984.

3. Bin, H., Inputting constructive solid geometry directly from $2 \mathrm{D}$ orthographic views, CAD,Vol. 18, No. 3, pp. 147-155, 1986.

4. Bowyer, A., Woodwark, J., A Programmers Geometry, Butterworths, London, England, 1985. 
5. Chen, Z. and Perng, D., Automatic reconstruction of 3D solid objects from 2D orthographic views, Pattern Recognition, Vol. 21, No. 5, pp. 439-449, 1988.

6. Dori, D., Liang, Y., Dowell, J. and Chai, I., Sparse pixel recognition of primitives in engineering drawings, Machine Vision and Applications, Vol. 6, pp. 69-82. 1993.

7. Dori, D., Representation pattern recognition-embedded systems through objectprocess diagrams: the case of machine drawing understanding system, Pattern recognition letters, Vol. 16, pp. 377-384, 1995.

8. Dori, D., Dimensioning analysys: a step toward automatic high level of understanding of engineering drawings, Comm. of the ACM, Vol. 35, pp. 92103, 1992.

9. Gujar. U. G. and Nagendra, I. V., Construction of 3D solid objects from orthographic views, Computers and graphics, Vol. 13, No. 4, pp. 502-521, 1989.

10. Lequelte, R., Automatic construction of curvilinear solids from wireframe views, CAD. Vol. 20, No. 4, pp. 171-180, 1988.

11. Markowsky, G. and Wesley, M. A., Fleshing out wireframes, IBM J. of Research and Development, Vol. 24, No. 5, pp. 582-597, 1980.

12. Martson, R. E. and Kuo, M. H., Reconstruction of 3D object from three orthographic projections using a decision chaining method, IAPR MVA'94, Workshop on Machine Vision Applications, Kawasaki, Japan, 13-15 Dec. 1994.

13. Meeran. S. and Pratt, M. J., Automated feature recognition from 2D views, CAD, Vol. 25, No. 1, pp. 7-17, 1993.

14. Nagasami, V. and Noshir, A. L., Reconstructing of three-dimensional object using a knowledge-based environment, Enginecring with Computers, Vol. 7, pp. 23-35, 1991.

15. Sakurai, H. and Gossard, D. C., Solid model input through orthographic views, Computer Graphics (SIGGRAPH), Vol. 17, No. 3, 1983.

16 Tombre, $K$. and Vaxiviere, P., Structure, syntax and semantics in technical document recognition, Proc. of the First International Conference on Document Analysis and Recognition. IEEE Computer Society, Saint-Malo, France,1991.

17. Wesley, M. A. and Markowsky, G., Fleshing out projections, IBM J. of Research and Development, Vol. 25, No. 6, pp. 934-953, 1981. 